\title{
Durabilidad natural de madera de Eucalyptus grandis Hill ex Maiden de plantaciones de rápido crecimiento*
}

\author{
Böthig, S. (1), Sánchez, A. (1), Doldán, J. (1) \\ Contacto: sbothig@latu.org.uy \\ (1) Departamento de Proyectos Forestales, Laboratorio Tecnológico del Uruguay (LATU) \\ Recibido: 15/9/2008 - Aprobado: 31/12/2008
}

*Ponencia presentada en la 39th Annual Meeting of International Research Group on Wood Protection, (Estambul 25-29 de mayo de 2008). Estambul: IRG, 2008 .

\begin{abstract}
$\underline{\text { Resumen }}$
Una de las especies forestales de rápido crecimiento cultivadas en Uruguay de mayor importancia económica es el Eucalyptus grandis. Trabajos anteriores reportan propiedades físicas y mecánicas de la madera juvenil y adulta proveniente de plantaciones de esta especie en diferentes regiones del país. Sin embargo, dado que no se dispone de datos científicos nacionales sobre su durabilidad natural, en este trabajo fue evaluada madera de $\mathbf{E}$. grandis obtenida de dos plantaciones de 16 años de edad con semilla del mismo origen, de dos sitios, Rivera y Río Negro. Se estudió la durabilidad natural del duramen externo e interno siguiendo los métodos de la norma EN 350-1, tomando Populus deltoides x euroamericana cv I-214 como especie de referencia. Se realizaron ensayos de laboratorio para determinar la resistencia a la descomposición fúngica (Gloeophyllum trabeum, Trametes versicolor y Serpula lacrymans) y a las termites (Reticulitermes spp.) siguiendo las normas EN 113 y EN 118, respectivamente. Se realizaron ensayos de campo de estacas, de doble capa y cámara fúngica, los cuales aún están en curso, por lo que en el presente trabajo se presentan resultados parciales. Los perfiles radiales de densidad básica revelaron que la madera del duramen externo no era adulta, sino madera de transición. En general, el duramen de E. grandis mostró una mayor durabilidad que el híbrido Populus. El E. grandis se clasificó como moderadamente o seriamente atacado por Reticulitermes spp. Según EN 350-1, la madera juvenil de E. grandis puede considerarse como "moderadamente durable" ante la pudrición parda provocada por G. trabeum, mientras que la madera de transición puede describirse como "durable". La madera de transición mostró en relación a la madera juvenil una mayor resistencia al G. trabeum, una susceptibilidad levemente menor a las termites y mejor desempeño en el campo luego de 17 meses de exposición. El sitio de la plantación no parece afectar la vulnerabilidad a las termites del E. grandis cultivado en la región norte u oeste de Uruguay. Hasta el momento, los resultados de campo sugieren mejor desempeño para el sitio II que para el sitio I. Sin embargo, aún es muy pronto para extraer conclusiones válidas de los ensayos de doble capa o de estacas. La durabilidad natural podría estar relacionada con la densidad. Otros estudios son necesarios para extraer conclusiones certeras al respecto.
\end{abstract}

Palabras clave: Eucalyptus grandis, durabilidad natural, madera juvenil, densidad.

\begin{abstract}
One of the fast growing species cultivated in Uruguay of major economical importance is Eucalyptus grandis. Physical and mechanical properties of juvenile and mature wood from different regions have been reported. However, since no scientific data of natural durability of this specie has been reported, natural durability of E. grandis from two sites (Rivera and Río Negro) was evaluated. Two sixteen years old plantations, originated by seed of same source, were sampled. Natural durability of outer and inner heartwood was studied following EN 350-1 methods. Populus deltoides x euroamericana cv I-214 was tested as reference specie. EN 113 laboratory tests for fungal decay (Gloeophyllum trabeum, Trametes versicolor and Serpula lacrymans) and EN 118 for termites resistance (Reticulitermes spp.) were performed. Field stakes, double layer and fungal cellar tests were established. They are still on course and partial results are reported below in this paper. Upon basic density radial profiles, it was found that outer heartwood was not mature wood, but can be assumed as transition wood. In general, E. grandis heartwood showed higher durability than Populus hybrid. E. grandis appeared moderately or seriously attacked by Reticulitermes spp. E. grandis juvenile wood can be classified according EN 350-1, as "Moderately Durable" against the brown rotter G. trabeum, whereas transition wood can be described as "Durable". Transition wood has shown higher resistance to G. trabeum, slightly less susceptibility to termites and a better condition in the field (17 months exposure) than juvenile wood. Site of plantation does not seem to affect termites susceptibility of E. grandis cultivated in the Northern or Western region of Uruguay. Field performance seems to be slightly better for site II than site I. However, it is too soon to draw valid conclusions from double layer or field stakes test. Natural durability might be related to density. Deeper studies should be done to draw any certain conclusion.

Key words: Eucalyptus grandis, natural durability, juvenile wood, wood density.
\end{abstract}

\section{Introducción}

Eucalyptus grandis Hill ex Maiden es una de las especies forestales más importantes plantadas con destino de aserrío en Uruguay. Si bien existen varios estudios de investigación sobre sus propiedades físicas y mecánicas (Doldán, 2001; Pérez del Castillo, 2001; O’Neill et al. 2005a, 2005b y 2005c), existe escasa información sobre la durabilidad natural del E. grandis cultivado (Da Silva Oliveira, 1999), y ninguna disponible sobre el duramen de $E$. grandis proveniente de plantaciones de rápido crecimiento en Uruguay. Por lo tanto, y considerando que el duramen es sumamente difícil de impregnar, se decidió estudiar su durabilidad.

Se ha observado variación en las tasas de crecimiento, así como 
en la calidad de la madera entre las diferentes regiones forestales en Uruguay. Si bien Malan (1997) indica que no existe una correlación significativa entre la tasa de crecimiento y la calidad de la madera de E. grandis, establece un considerable efecto del sitio en la calidad, que se manifiesta, por ejemplo, en la densidad y la longitud de fibras.

Al no disponerse al momento de realizar este trabajo de plantaciones clonales, se evaluó el efecto del sitio en algunas propiedades físicas y la durabilidad natural, estudiando madera de dos montes originados de la misma semilla, plantados en el mismo año y cosechados a la misma edad. Los dos montes escogidos tenían tasas de crecimiento típicamente diferentes.

Es un hecho conocido que la madera adulta de E. grandis tiene propiedades de mejor calidad que la madera juvenil, tales como mayor densidad y mayor resistencia mecánica (Doldán, 2001; Pérez del Castillo, 2001; Doldán y Böthig, 2002). A pesar de que esta diferencia es menos marcada que en las coníferas, es necesario tenerla en cuenta, especialmente para la gestión y la cosecha forestal. Esta especie de árbol de rápido crecimiento se cosecha generalmente a edades muy tempranas, por lo que el estudio de madera juvenil parece ser un punto importante para la cadena de transformación de la madera.

El presente trabajo propone evaluar las diferencias en la durabilidad entre la madera juvenil y adulta y la posible relación con la densidad.

El estudio, que aún se está desarrollando en base a la norma EN 350-1, incluye ensayos de laboratorio para determinar el deterioro debido a la acción de hongos y termites. También abarca las siguientes pruebas de campo: ensayo de estacas y de doble capa y el ensayo de cámara fúngica (EN 252; Rapp et al. 2001; Nagano, 2001).

Como especie de referencia se estudió Populus deltoides $x$ euroamericana cv I-214 (álamo). Este género de madera se considera como clase 5, "no durable", según la categorización EN 350-2, y se comporta de forma similar a la haya (Van Acker et al. 2003). A diferencia de Fagus sylvatica (haya), esta especie se planta en Uruguay y su madera seca al aire puede adquirirse fácilmente en el mercado.

Este trabajo forma parte de un proyecto de investigación titulado "Durabilidad y preservación de las maderas nacionales para uso en la construcción" (PDT S/C/OP/35-11), que se está desarrollando en el LATU desde setiembre de 2006, apoyado por el gobierno (CONICYT) y el LATU.

\section{Materiales y Métodos}

\section{Madera de Eucalyptus grandis}

Se obtuvieron muestras de dos plantaciones originadas de la misma semilla local de E. grandis Hill Ex Maiden en diferentes lugares del país.

- Semilla: Bañados Medina (local).

- Edad al momento de la cosecha: 16.

- Sitio I: Rivera, Uruguay (norte).

- Ubicación geográfica: LS $31^{\circ} 21^{\prime} 15.79^{\prime \prime}$ - LO 55² 27'31.34",

- Densidad de la plantación al momento del muestreo: 130 árbol/ha.

- Sitio II: Río Negro, Uruguay (Oeste).

- Ubicación geográfica: LS $32^{\circ} 54^{\prime} 41.67$ "- LO 57 $58^{\circ}$ ' 48.25".

- Densidad de la plantación al momento del muestreo: 756 árbol/ha.

Muestreo. Se seleccionaron 50 árboles en función de la forma del fuste, el estado sanitario y la forma de la copa. Se midieron el diámetro y la penetración por Pilodyn a la altura del pecho. De acuerdo a la distribución natural del diámetro y Pilodyn se seleccionaron los individuos a muestrear. Número de árboles muestreados: 8 (sitio I) y 10 (sitio II). En la Tabla 1 se presentan las características de la muestra obtenida.

Proceso de aserrado. Los árboles fueron procesados en el aserradero del laboratorio dentro de los 10 días después de ser cosechados.

De este proceso se obtuvieron listones de 2 pulgadas de ancho, que se distribuyeron en las siguientes clases: tablas de albura, tablas de duramen interno denominadas "A" (alrededor de la médula) y tablas de duramen externo denominadas " $\mathrm{C}$ ".

\begin{tabular}{|l|c|c|c|c|}
\hline \multirow{2}{*}{} & \multicolumn{2}{|c|}{ SITIO I } & \multicolumn{2}{c|}{ SITIO II } \\
\cline { 2 - 5 } & $\begin{array}{c}\text { DAP } \\
(\mathrm{cm})\end{array}$ & $\begin{array}{c}\text { Pilodyn } \\
(\mathrm{mm})\end{array}$ & $\begin{array}{c}\text { DAP } \\
(\mathrm{cm})\end{array}$ & $\begin{array}{c}\text { Pilodyn } \\
(\mathrm{mm})\end{array}$ \\
\hline Media & 44,3 & 16,0 & 36,3 & 15,0 \\
\hline Mín. & 39,0 & 14,0 & 29,0 & 13,5 \\
\hline Máx. & 54,7 & 18,0 & 42,0 & 16,5 \\
\hline CV(\%) & 12 & 8,0 & 12 & 6,7 \\
\hline
\end{tabular}

Tabla 1. Valores del DAP y penetración por Pilodyn de los árboles muestreados en ambos sitios.

Secado. Inmediatamente después del aserrado, la madera se secó en horno, siguiendo programas de baja temperatura. La madera del sitio I fue procesada en un horno de secado convencional, mientras que por razones locativas la madera del sitio II debió secarse en un horno de secado solar.

\section{Madera de referencia}

Se utilizó madera de Populus deltoides $x$ euroamericana $c v$ I.214 proveniente de plantaciones del departamento de San José, en la región sur del país. No se dispone de datos de la plantación. Se seleccionaron tablas secadas al aire de diferentes pilas extraídas de más de tres árboles.

\section{Densidad básica}

De cada troza se muestreó una rodaja a una altura de aproximadamente $2,60 \mathrm{~m}$. Se cortó una tira de $4 \mathrm{~cm}$ de ancho sin defectos, a lo largo de todo el diámetro. Luego se cortó en trozos desde la médula a la corteza de forma regular. Se calculó la densidad básica como el cociente entre el peso anhidro y el volumen saturado (ASTM D 2395).

\section{Acondicionamiento}

Todas las muestras se mantuvieron en una cámara de acondicionamiento a $20^{\circ} \mathrm{C}$ y $65 \% \mathrm{HR}$ hasta la ejecución de los ensayos biológicos de laboratorio.

\section{Ensayos de laboratorio para determinar la resistencia a la descomposición fúngica}

Se siguieron los procedimientos de la norma EN 113 y se consideraron cuatro niveles:

- Sitio I, posición A

- Sitio I, posición C

- Sitio II, posición A

- Sitio II, posición C

En el presente estudio se incluyeron las tres especies de hongos basidiomicetes recomendados en la norma EN 350-1 para los ensayos de madera de latifoliadas:

Trametes versicolor (ATCC 12679),

Gloeophyllum trabeum (ATCC11539),

Serpula lacrymans (BAM Ebw 315).

Se realizó la incubación en cámara acondicionada a $22{ }^{\circ} \mathrm{C}$ durante 
16 semanas. Para los ensayos del sitio I se mantuvo la humedad relativa (HR) con una solución saturada de cloruro sódico. En el caso de las pruebas del sitio II, se controló la HR a $70 \%$.

Al finalizar los ensayos se registraron las descripciones visuales del micelio, del medio de cultivo y de la probeta, así como del crecimiento del micelio en la misma. Se evaluó el deterioro en forma cualitativa y se cuantificó el contenido de humedad final y la pérdida de peso.

\section{Ensayos de laboratorio para determinar la resistencia al ataque de termites}

Fueron realizados de acuerdo con la norma EN 118 (Wood preservatives - Determination of preventive action against Reticulitermes species, 2005). Se recogieron termites del género Reticulitermes spp. ${ }^{2}$ alrededor de las instalaciones del LATU y se formaron siete colonias, que fueron mantenidas en laboratorio. De estas colonias se tomaron las termites para los ensayos. Las colonias se armaron de forma tal que todos los individuos provinieran de la misma colonia madre.

Se seleccionaron cinco árboles de cada sitio para la prueba. Se evaluaron duplicados de cada posición radial (A y C) para cada árbol. Tanto la posición A como $\mathrm{C}$ se evaluó usando termites que provenían de una misma colonia. Los ensayos de albura de E. grandis y del híbrido Populus aún se están desarrollando.

El número de soldados y ninfas se determinó según la proporción natural de las castas presentes en las colonias.

Todos los ensayos transcurrieron durante ocho semanas bajo condiciones estándar. Se supervisaron diariamente para mantener el contenido de humedad y realizar control de fugas. Cada especimen se mantuvo en una caja de plástico y de esta forma los individuos escapados se devolvieron al ensayo. Los insectos muertos fueron sustituidos por individuos de las colonias. Se sellaron las perforaciones, se observó y se registró la actividad de la colonia, se destruyeron las chimeneas y túneles externos.

Luego de ocho semanas, se hizo un recuento de los sobrevivientes y se clasificó el daño a la madera según la escala de clasificación de la norma EN 118. Se evaluaron el contenido de humedad y la pérdida de peso.

\section{Ensayo de campo con estacas}

Se prepararon estacas de las tablas A y C de todos los árboles, según la norma EN 252 (Field test method for determining the relative protective effectiveness of a wood preservative in ground contact, 1989).

Se ensayó el híbrido Populus como especie de referencia. El ensayo se realizó en los alrededores del laboratorio, en donde existen poblaciones activas de termites. En este predio se encontraron dos especies, y si bien una corresponde al género Reticulitermes, ambas están en proceso de identificación.

Luego de transcurrido un año y medio desde la instalación del ensayo, algunas estacas debieron ser sustituidas por sufrir daños externos. Se realizaron las evaluaciones según la norma EN 252, a partir de los 6,7 y 17,4 meses de exposición a la intemperie.

\section{Ensayo de doble capa}

El ensayo se diseñó según el método propuesto por Rapp et al. (2001, 2003a, 2003b, 2006). Consiste en un ensayo de exposición a la intemperie en posición horizontal sin contacto con el suelo. Las probetas se disponen paralelamente entre sí, en dos capas alternadas, formando un piso que se apoya por los extremos de las mismas sobre vigas de madera. Se prepararon probetas de las dimensiones establecidas por la norma EN 252 para la posición A y C de todos los árboles en ambos sitios.

Hasta el momento el ensayo lleva 17 meses desde su instalación.

\section{Densidad y contenido de humedad}

Se determinaron la densidad y el contenido de humedad para cada ensayo biológico y para cada probeta evaluada. A partir de listones se cortaron las probetas de ensayo y otras para determinar la densidad y el contenido de humedad.

Se calculó la densidad como el cociente del peso anhidro sobre el volumen en condiciones de ensayo. Las dimensiones de las probetas se midieron con un calibre digital (Mitutoyo). El contenido de humedad se determinó por el método de secado en estufa.

\section{Resultados y Discusión}

\section{Densidad básica}

Se realizó una prueba t de Student sobre valores experimentales y se confirmó que las densidades de la madera de ambos sitios eran diferentes, presentando el sitio II valores más altos que los del sitio I

\begin{tabular}{|c|c|c|}
\hline & Sitio I & Sitio II \\
\hline Media & 0,390 & 0,464 \\
\hline Mín. & 0,327 & 0,414 \\
\hline Máx. & 0,513 & 0,530 \\
\hline N & 42 & 40 \\
\hline Std & 0,038 & 0,028 \\
\hline CV (\%) & 9,8 & 6,1 \\
\hline
\end{tabular}

$\mathrm{N}$ = número de repeticiones; $\mathrm{Std}=$ desviación estándar; $\mathrm{CV}=$ coeficiente de variación

Tabla 2. Densidad básica (g/cm3). Comparación de ambos sitios ${ }^{3}$.

Las Figuras 1 y 2 muestran la distribución radial de la densidad básica para cada árbol. Se evidencia que los individuos siguen patrones diferentes, pero la tendencia general es el aumento con la distancia relativa desde la médula. No hay evidencia clara de la estabilización de las pendientes de las curvas para todos los árboles, lo que significa que es posible que los tejidos externos sigan siendo madera de transición (Downs, 1997). Esto concuerda con las zonas de transición ya indicadas por Doldán y Böthig (2002) y Doldán (2003). Para confirmar lo anterior, deberían realizarse análisis más detallados de los datos experimentales y estudios adicionales tales como medidas de la longitud de fibras.

El último punto de cada perfil corresponde a la albura. Esto reafirma la idea de que el duramen externo estudiado aquí sería madera de transición.

2 La identificación de la especie aún está siendo realizada por la Dra. Ana Aber, Facultad de Ciencias, UDELAR, Universidad de la República Oriental del Uruguay.

3 Los datos de los $10 \mathrm{~cm}$ internos (en diámetro) del rolo fueron excluidos. 


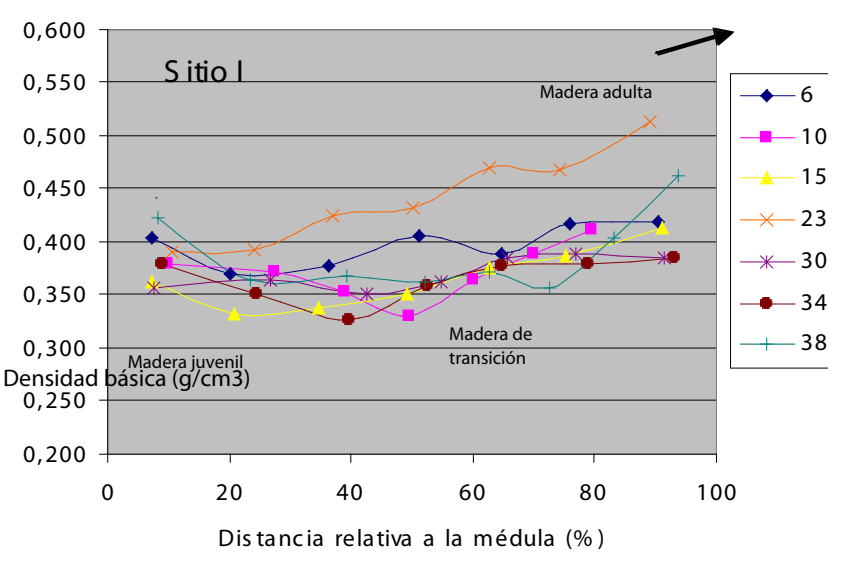

Figura 1. Perfiles radiales individuales de la densidad básica para cada árbol. Sitio I.

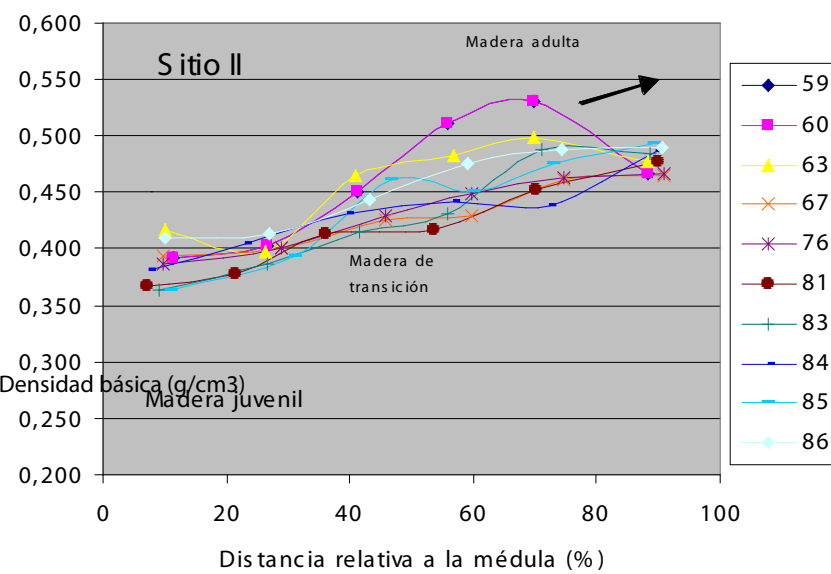

Figura 2. Perfiles radiales individuales de la densidad básica para cada árbol. Sitio II.

Todos los resultados se expresan como porcentaje y se corrigen por la pérdida de peso de los controles. Se indican los valores de la mediana, ya que se considera que disminuyen el impacto de los valores extremos (Van Acker et al. 2003). En los resultados de estos ensayos se evidencia que el promedio resulta sumamente afectado por los valores extremos, mientras que la mediana refleja mejor la tendencia general. Se aplicaron pruebas $t$ de Student con $\alpha=0,05$ para la comparación de las medias.

Trametes versicolor. Todos los hongos evolucionaron de forma adecuada durante las pruebas, cubriendo completamente la superficie del medio y no resultaron afectados por ningún tipo de contaminación. Sin embargo, Trametes versicolor presentó una virulencia extremadamente baja en ambas pruebas (A y C), tanto para el sitio I como para el sitio II. Esto se reflejó en los resultados de la pérdida de peso: un valor promedio bajo y un coeficiente de variación sumamente alto (Tablas 3 y 4). La pérdida de masa no cumplió con los requisitos de la norma ni coincidió con los valores de la referencia bibliográfica (Van Acker et al. 2003 y EN 350-2).

No es posible extraer mayores conclusiones de estos resultados debido a la baja virulencia demostrada por la cepa utilizada.

No obstante, es posible señalar que bajo las condiciones especiales descritas anteriormente, el E. grandis presentó mayor resistencia que el álamo a la descomposición de T.versicolor.

\begin{tabular}{|c|c|c|c|c|c|c|}
\hline Especie & \multicolumn{2}{|c|}{ E. grandis } & \multicolumn{2}{|c|}{ Álamo } & \multicolumn{2}{|c|}{$\begin{array}{c}\text { Relación } \\
\text { Eg/álamo }\end{array}$} \\
\hline $\begin{array}{c}\text { Posición } \\
\text { radial }\end{array}$ & $\mathbf{A}$ & $\mathbf{C}$ & $\mathbf{A}$ & $\mathbf{C}$ & $\mathbf{A}$ & $\mathbf{C}$ \\
\hline MEDIA & 1,86 & 3,42 & 11,02 & 9,62 & 0,41 & 11,50 \\
\hline MEDIANA & 1,73 & 2,87 & 8,36 & 4,49 & 0,22 & 0,68 \\
\hline MÍN. & 0,26 & 0,60 & 1,92 & $-0,07$ & 0,01 & 0,03 \\
\hline MÁX. & 3,8 & 7,70 & 26,5 & 26 & 1,99 & 75 \\
\hline N & 8 & 8 & 8 & 8 & 8 & 8 \\
\hline STD & 1,1 & 2,5 & 7,9 & 10,7 & 0,70 & 26,0 \\
\hline CV & 61 & 73 & 71 & 112 & 170 & 226 \\
\hline
\end{tabular}

Eg/álamo = pérdida de peso relativa calculada para los pares de cada recipiente de ensayo.

Tabla 3. Resultados de la pérdida de peso para EN 113 (\%): Sitio I / Trametes versicolor.

\begin{tabular}{|c|c|c|c|c|c|c|}
\hline \multirow{2}{*}{ Especie } & \multicolumn{2}{|c|}{ E. grandis } & \multicolumn{2}{|c|}{ Álamo } & \multicolumn{2}{c|}{$\begin{array}{c}\text { Relación } \\
\text { Eg/álamo }\end{array}$} \\
\hline Posición radial & A & C & A & C & A & C \\
\hline MEDIA & 11,1 & 4,50 & 7,29 & 5,88 & 40,0 & 14,7 \\
\hline MEDIANA & 7,76 & 4,74 & 3,82 & 3,25 & 3,57 & 1,56 \\
\hline MÍN. & 2,96 & 0,31 & $-0,35$ & $-0,09$ & 0,177 & 0,019 \\
\hline MÁX. & 27,3 & 12,9 & 20,9 & 19,9 & 165 & 90 \\
\hline N & 10 & 10 & 10 & 10 & 10 & 10 \\
\hline STD & 8,8 & 3,9 & 8,2 & 7,6 & 60 & 30 \\
\hline CV & 79 & 87 & 113 & 129 & 151 & 202 \\
\hline
\end{tabular}

Tabla 4. Resultados de la pérdida de peso para EN 113 (\%): Sitio II / Trametes versicolor.

En consecuencia, se tomaron dos medidas:

- Para aumentar la virulencia del inóculo para ensayos posteriores, se inoculó la misma cepa en madera de Eucalyptus previamente incorporada en agar malta, incubada a $22{ }^{\circ} \mathrm{C}$ y luego transferida a placas de Petri con agar malta;

- Se analizó la descomposición por T. versicolor en el Populus y la albura de Eucalyptus conjuntamente.

La Tabla 5 muestra cómo mejoró significativamente la virulencia de T. versicolor. La mediana de la pérdida de peso del álamo aumentó de cerca de $10 \%$ hasta $41 \%$. El coeficiente de variación disminuyó de aproximadamente $100 \%$ a $33 \%$. Según la prueba t de Student, no es posible encontrar diferencias entre las pérdidas de peso de la albura E. grandis y el álamo.

\begin{tabular}{|c|c|c|c|}
\hline Especie & E. grandis & Álamo & $\begin{array}{c}\text { Relación } \\
\text { Eg/álamo }\end{array}$ \\
\hline MEDIA & 48,0 & 44,0 & 1,26 \\
\hline MEDIANA & 45,9 & 41,2 & 1,05 \\
\hline MÍN. & 36,7 & 26,3 & 0,61 \\
\hline MÁX. & 63,4 & 62,2 & 2,41 \\
\hline N & 6 & 6 & 6 \\
\hline STD & 11,4 & 14,5 & 0,7 \\
\hline CV & 24 & 33 & 56 \\
\hline
\end{tabular}

Tabla 5. Resultados de la pérdida de peso para EN 113 (\%): duramen de E. grandis y álamo / Trametes versicolor.

La misma cepa analizada en Pinus taeda arrojó pérdidas de masa de hasta $60 \%{ }^{4}$

4 Datos aún no publicados. 
Gloeophyllum trabeum. Las medias de la pérdida de masa para álamo fueron comparadas para los ensayos de ambos sitios y posiciones mediante pruebas t de Student, no mostrando diferencia estadística significativa. Esto significa que los cuatro ensayos pueden compararse unos con otros. Se calcula el promedio de todas las pérdidas de peso del álamo, que alcanza aproximadamente un $20 \%$. En consecuencia, los resultados que figuran en la Tabla 6 se consideraron válidos de acuerdo a lo establecido por la norma, a pesar de ser más bajos que los valores reportados en otros trabajos (Van Acker et al. 2003 y EN 350-2). La misma cepa analizada posteriormente en Pinus taeda en el laboratorio causó pérdidas de masa de entre 30 y $60 \%{ }^{3}$.

Si se utiliza el valor de la mediana de la relación Eg/álamo, la madera juvenil de E. grandis (posición A) resultó "moderadamente durable" según la clasificación de EN 350-1, mientras que la madera de transición (posición C) se incluyó en la clase "durable". Se evaluó la pérdida de masa absoluta según los criterios de clasificación propuestos por Van Acker et al (2003). La madera de transición de ambos sitios pudo clasificarse como "durable" ante G. trabeum. Mientras que la madera juvenil del sitio I se incluyó en la misma categoría, la madera juvenil del sitio II resultó en la clase "moderadamente durable".

\begin{tabular}{|c|c|c|c|c|c|c|}
\hline Especie & \multicolumn{2}{|c|}{ E. grandis } & \multicolumn{2}{c|}{ Álamo } & \multicolumn{2}{c|}{$\begin{array}{c}\text { Relación } \\
\text { Eg/álamo }\end{array}$} \\
\hline $\begin{array}{c}\text { Posición } \\
\text { radial }\end{array}$ & A & C & A & C & A & C \\
\hline MEDIA & 7,30 & 5,85 & 21,8 & 18,0 & 1,01 & 3,63 \\
\hline MEDIANA & 6,30 & 5,97 & 24,7 & 20,8 & 0,35 & 0,29 \\
\hline MÍN. & 4,61 & 3,08 & 1,73 & 0,30 & 0,11 & 0,13 \\
\hline MÁX. & 12,7 & 9,10 & 42,1 & 39,7 & 5,78 & 14,1 \\
\hline N & 8 & 8 & 8 & 8 & 8 & 8 \\
\hline STD & 3,0 & 1,9 & 13,1 & 14,3 & 2,1 & 6,0 \\
\hline CV & 41 & 33 & 60 & 80 & 203 & 165 \\
\hline
\end{tabular}

Tabla 6. Resultados de la pérdida de peso para EN 113 (\%): Sitio I / Gloeophyllum trabeum

\begin{tabular}{|c|c|c|c|c|c|c|}
\hline Especie & \multicolumn{2}{|c|}{ E. grandis } & \multicolumn{2}{|c|}{ Álamo } & \multicolumn{2}{c|}{$\begin{array}{c}\text { Relación } \\
\text { Eg/álamo }\end{array}$} \\
\hline $\begin{array}{c}\text { Posición } \\
\text { radial }\end{array}$ & $\mathbf{A}$ & $\mathbf{C}$ & $\mathbf{A}$ & $\mathbf{C}$ & $\mathbf{A}$ & $\mathbf{C}$ \\
\hline MEDIA & 9,57 & 4,82 & 17,0 & 22,1 & 1,25 & 14,3 \\
\hline MEDIANA & 10,13 & 4,97 & 17,7 & 21,8 & 0,57 & 0,15 \\
\hline MÍN. & 0,78 & 1,30 & 2,44 & $-0,06$ & 0,14 & 0,064 \\
\hline MÁX. & 16,0 & 8,05 & 38,7 & 45,3 & 4,77 & 113 \\
\hline N & 10 & 10 & 10 & 10 & 10 & 10 \\
\hline STD & 4,11 & 2,32 & 13,1 & 14,1 & 1,48 & 35,8 \\
\hline CV & 43 & 48 & 77 & 64 & 118 & 250 \\
\hline
\end{tabular}

Tabla 7. Resultados de la pérdida de peso para EN 113 (\%): Sitio II / Gloeophyllum trabeum.

Se observan menores pérdidas de peso al aumentar la densidad; sin embargo, el coeficiente de correlación no es significativo (Figura 3).

Como el contenido de los extractivos en la madera puede afectar la durabilidad natural, deberían ensayarse probetas previamente extraídas para eliminar la incidencia de este parámetro. Esto permitiría evaluar más claramente el efecto de la densidad sobre la durabilidad, eliminando el efecto de los extractivos.

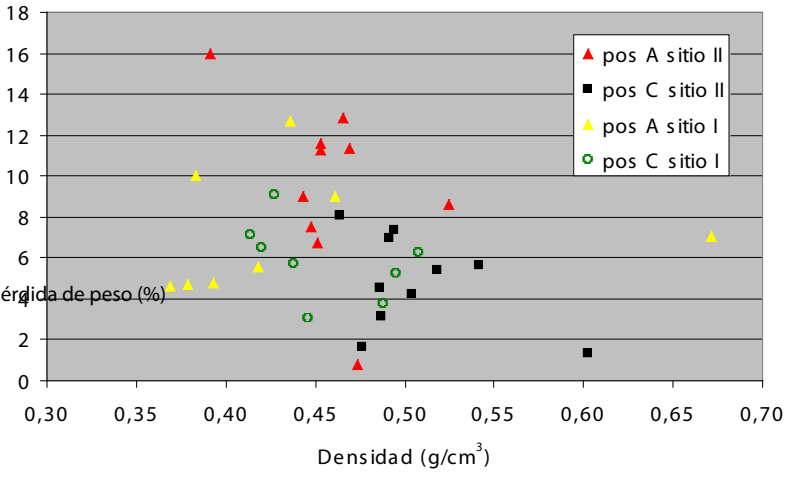

Figura 3. Pérdida de peso del duramen de $E$. grandis representada en función de la densidad de la madera.

Serpula lacrymans. Si bien se observó crecimiento vigoroso del micelio sobre el medio de cultivo, en general no colonizó especímenes de álamo. No obstante, la colonización tuvo éxito en los bloques de E. grandis, resultando más vigorosa en los especimenes del sitio I. De todas formas, la pérdida de peso no correlacionó con este crecimiento (Tablas 8 y 9 ).

\begin{tabular}{|c|c|c|c|c|c|c|}
\hline Especie & \multicolumn{2}{|c|}{ E. grandis } & \multicolumn{2}{|c|}{ Álamo } & \multicolumn{2}{c|}{$\begin{array}{c}\text { Relación } \\
\text { Eg/álamo }\end{array}$} \\
\hline $\begin{array}{c}\text { Posición } \\
\text { radial }\end{array}$ & A & C & A & C & A & C \\
\hline MEDIA & 3,54 & 1,63 & 2,20 & 0,36 & 7,47 & 4,13 \\
\hline MEDIANA & 2,53 & 1,73 & 0,48 & 0,39 & 5,01 & 3,07 \\
\hline MÍN. & 0,11 & $-0,04$ & 0,28 & 0,10 & 0,14 & $-0,16$ \\
\hline MÁX. & 15,1 & 3,98 & 14,2 & 0,58 & 35,8 & 10,9 \\
\hline N & 8 & 8 & 8 & 8 & 8 & 8 \\
\hline STD & 4,8 & 1,4 & 4,8 & 0,2 & 11,8 & 3,3 \\
\hline CV & 137 & 88 & 220 & 50 & 158 & 81 \\
\hline
\end{tabular}

Eg/álamo = pérdida de peso

relativa calculada para los pares de cada matraz.

Tabla 8. Resultados de la pérdida de peso para EN 113 (\%): Sitio I/ Serpula lacrymans.

\begin{tabular}{|c|c|c|c|c|c|c|}
\hline Especie & \multicolumn{2}{|c|}{ E. grandis } & \multicolumn{2}{c|}{ Álamo } & \multicolumn{2}{c|}{$\begin{array}{c}\text { Relación Eg/ } \\
\text { álamo }\end{array}$} \\
\hline $\begin{array}{c}\text { Posición } \\
\text { radial }\end{array}$ & A & C & A & C & A & C \\
\hline MEDIA & $-0,05$ & $-0,80$ & 0,33 & $-1,15$ & 74,20 & 60,30 \\
\hline MEDIANA & 0,41 & 0,12 & 0,20 & 0,23 & 3,81 & 0,67 \\
\hline MÍN. & $-5,19$ & $-3,62$ & 0,12 & $-14,30$ & 33,09 & 27,49 \\
\hline MÁX. & 2,9 & 0,16 & 0,8 & 1,56 & 101,2 & 106,5 \\
\hline N & 9 & 5 & 9 & 9 & 9 & 9 \\
\hline STD & 2,1 & 1,6 & 0,2 & 5,0 & 23,4 & 32,4 \\
\hline CV & -- & -- & -- & -- & 32 & 54 \\
\hline
\end{tabular}

Tabla 9. Resultados de la pérdida de peso para EN 113 (\%): Sitio II / Serpula lacrymans.

En base a los resultados arriba expuestos, se ensayó la descomposición por Serpula sobre Populus y albura de Eucalyptus conjuntamente, repitiéndose los resultados sobre álamo; un buen desarrollo sobre el medio de cultivo en todos los recipientes de ensayo, crecimiento vigoroso sobre los bloques de Eucalyptus y muy poco o ninguna colonización sobre álamo. No se encontró daño cualitativo o 
cuantitativo (Tabla 10).

\begin{tabular}{|c|c|c|c|}
\hline Especie & $\begin{array}{c}\text { Albura de } \\
\text { E. grandis }\end{array}$ & Álamo & $\begin{array}{c}\text { Relación } \\
\text { Eg/álamo }\end{array}$ \\
\hline MEDIA & $-0,07$ & $-0,18$ & $-0,07$ \\
\hline MEDIANA & $-0,04$ & $-0,18$ & $-0,04$ \\
\hline MÍN. & $-0,58$ & $-0,80$ & $-0,58$ \\
\hline MÁX. & 0,40 & 0,70 & 0,40 \\
\hline N & 6 & 6 & 6 \\
\hline STD & 0,31 & 0,66 & 0,31 \\
\hline
\end{tabular}

Tabla 10. Resultados de la pérdida de peso para EN $113(\%)$ : albura de E. grandis y álamo / Serpula lacrymans.

\section{Ensayos de laboratorio para termites}

La clasificación de los daños osciló entre 2 y 4 , con un valor de la media de 3,6 (Tabla 11, Figura 4). Esto significa vulnerabilidad moderada y alta vulnerabilidad del duramen de E. grandis al ataque de las termites (EN 118).

Al comparar la posición A con la $\mathrm{C}$ no existen diferencias estadísticas entre los valores de la media, pero es posible observar una menor frecuencia de la clasificación 4 para la posición C (en ambos sitios). Esto sugeriría que la vulnerabilidad ante termites podría ser más baja en los tejidos adultos.

Los resultados correspondientes a la madera del sitio II sugieren una menor vulnerabilidad que los del sitio I. A pesar de ello, no es posible encontrar diferencias estadísticas entre las pérdidas de peso.

\begin{tabular}{|c|c|c|c|c|c|c|}
\hline SITIO & $\begin{array}{c}\text { Posición } \\
\text { radial }\end{array}$ & & $\begin{array}{c}\text { Densidad } \\
\left(\mathrm{g} / \mathrm{cm}^{3}\right)\end{array}$ & $\begin{array}{c}\mathrm{CH} \text { inicial } \\
(\%)\end{array}$ & $\begin{array}{c}\text { CH final } \\
(\%)\end{array}$ & $\begin{array}{c}\text { Pérdida de peso } \\
(\%)\end{array}$ \\
\hline \multirow{12}{*}{ I } & \multirow{6}{*}{ A } & Promedio & 0,433 & 10,9 & 14,8 & 2,0 \\
\hline & & Mín. & 0,368 & 10,6 & 9,5 & 1,2 \\
\hline & & Máx. & 0,684 & 11,0 & 17,2 & 2,8 \\
\hline & & Std & 0,087 & 0,18 & 2,16 & 0,51 \\
\hline & & $\mathrm{CV}$ & 20 & 1,6 & 15 & 26 \\
\hline & & $\mathrm{N}$ & 11 & 11 & 11 & 11 \\
\hline & \multirow{6}{*}{$\mathrm{C}$} & Promedio & 0,433 & 10,9 & 14,8 & 2,0 \\
\hline & & Mín. & 0,368 & 10,6 & 9,5 & 1,2 \\
\hline & & Máx. & 0,684 & 11,0 & 17,2 & 2,8 \\
\hline & & Std & 0,087 & 0,18 & 2,16 & 0,51 \\
\hline & & $\mathrm{CV}$ & 20 & 1,6 & 15 & 26 \\
\hline & & $\mathrm{N}$ & 11 & 11 & 11 & 11 \\
\hline \multirow{12}{*}{ II } & \multirow{6}{*}{ A } & Promedio & 0,467 & 10,6 & 16,3 & 1,8 \\
\hline & & Mín. & 0,387 & 10,4 & 14,9 & 1,1 \\
\hline & & Máx. & 0,517 & 10,8 & 17,8 & 2,4 \\
\hline & & Std & 0,042 & 0,14 & 0,98 & 0,38 \\
\hline & & $\mathrm{CV}$ & 9,1 & 1,4 & 6,0 & 21 \\
\hline & & $\mathrm{N}$ & 10 & 10 & 10 & 10 \\
\hline & \multirow{6}{*}{$\mathrm{C}$} & Promedio & 0,504 & 10,7 & 16,1 & 1,4 \\
\hline & & Mín. & 0,458 & 10,4 & 12,0 & 0,9 \\
\hline & & Máx. & 0,561 & 10,8 & 17,3 & 1,9 \\
\hline & & Std & 0,031 & 0,12 & 1,68 & 0,30 \\
\hline & & $\mathrm{CV}$ & 6 & 1,1 & 10 & 21 \\
\hline & & $\mathrm{N}$ & 10 & 10 & 10 & 9 \\
\hline
\end{tabular}

Tabla 11. Datos iniciales de los especímenes de prueba, contenido de humedad final y pérdida de peso. 


\begin{tabular}{|c|c|c|c|c|c|c|c|}
\hline \multirow{2}{*}{ SITIO } & \multirow{2}{*}{$\begin{array}{l}\text { Posición } \\
\text { radial }\end{array}$} & & \multirow{2}{*}{$\begin{array}{c}\mathrm{N}^{\mathrm{o}} \mathrm{de} \\
\text { perforaciones }\end{array}$} & \multirow{2}{*}{ Clasificación" } & \multicolumn{2}{|c|}{ Sobrevivientes $\left(\mathbf{N}^{\circ}\right)$} & \multirow{2}{*}{ Sobrevivientes (\%) } \\
\hline & & & & & Obreras & Soldados & \\
\hline \multirow{12}{*}{ I } & \multirow{6}{*}{ A } & Promedio & 0,64 & 3,9 & 76 & 4 & 35 \\
\hline & & Mín. & 0 & 3 & 33 & 2 & 15 \\
\hline & & Máx. & 3 & 4 & 99 & 6 & 44 \\
\hline & & Std & 0,92 & 0,30 & 19 & 1,4 & 8,6 \\
\hline & & $\mathrm{CV}$ & 145 & 7,7 & 25 & 37 & 25 \\
\hline & & $\mathrm{N}$ & 11 & 11 & 11 & 11 & 11 \\
\hline & \multirow{6}{*}{$\mathrm{C}$} & Promedio & 0 & 3,5 & 63 & 2 & 28 \\
\hline & & Mín. & 0 & 3 & 7 & 0 & 3 \\
\hline & & Máx. & 0 & 4 & 111 & 3 & 50 \\
\hline & & Std & 0 & 0,53 & 40 & 1,0 & 17,6 \\
\hline & & $\mathrm{CV}$ & --- & 15,1 & 63 & 57 & 62 \\
\hline & & $\mathrm{N}$ & 10 & 10 & 10 & 10 & 10 \\
\hline \multirow{12}{*}{ II } & \multirow{6}{*}{ A } & Promedio & 0,20 & 3,7 & 52 & 1 & 23 \\
\hline & & Mín. & 0 & 2 & 0 & 0 & 0 \\
\hline & & Máx. & 1 & 4 & 112 & 4 & 50 \\
\hline & & Std & 0,42 & 0,67 & 39 & 1,3 & 17,5 \\
\hline & & $\mathrm{CV}$ & 211 & 18,2 & 75 & 96 & 75 \\
\hline & & $\mathrm{N}$ & 10 & 10 & 10 & 10 & 10 \\
\hline & \multirow{6}{*}{$\mathrm{C}$} & Promedio & 0 & 3,2 & 49 & 3 & 22 \\
\hline & & Mín. & 0 & 2 & 0 & 0 & 0 \\
\hline & & Máx. & 0 & 4 & 97 & 5 & 44 \\
\hline & & Std & 0 & 0,79 & 35 & 2,2 & 15,8 \\
\hline & & $\mathrm{CV}$ & --- & 24,7 & 71 & 80 & 70 \\
\hline & & $\mathrm{N}$ & 10 & 10 & 10 & 10 & 10 \\
\hline
\end{tabular}

*Escala de clasificación: 0: sin daños, 1: tentativa de ataque, 2: daño leve, 3: daño moderado, 4: daño serio Tabla 12. Evaluación final de los ensayos: clasificación EN 118, perforaciones en madera y sobrevivientes.

Al analizar los resultados individuales se observa una buena repetibilidad. Además, algunos árboles parecerían tener una resistencia levemente superior (Figuras 4 y 5 ).

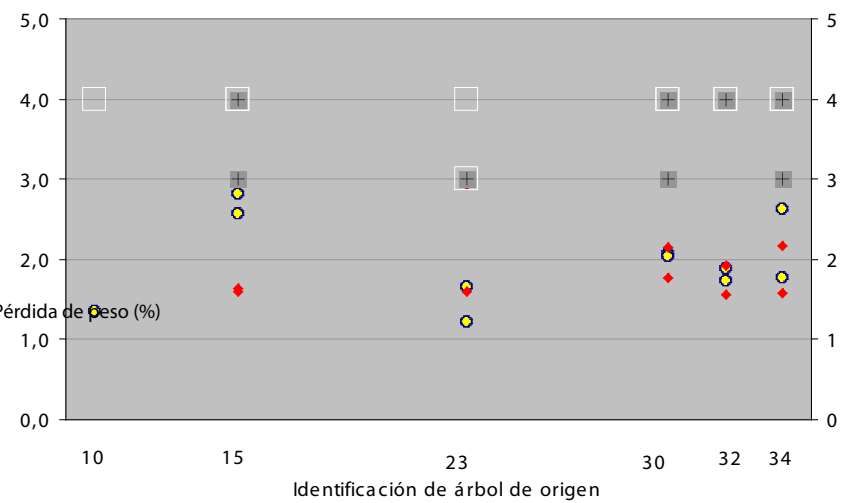

pérdida de peso- pos $A$ - pérdida de peso-pos $C$

clasificación - pos A + clasificación - pos $C$

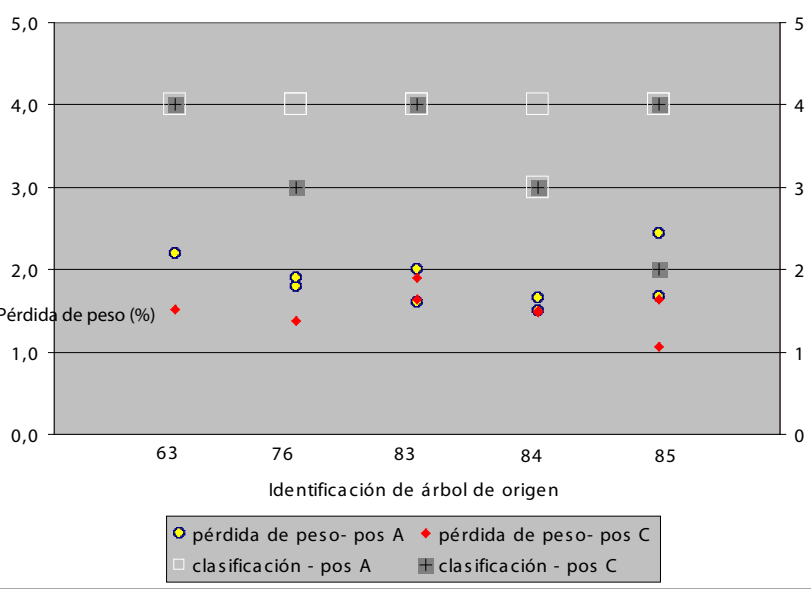

Figura 5. Resultados individuales de la pérdida de peso y clasificación según EN 118 representados en función de la identificación del árbol. Posiciones A y C. Sitio II.

A medida que aumenta la densidad de la madera se manifiesta una tendencia negativa para el ataque de las termites. Sin embargo, el coeficiente de correlación es bajo, especialmente en el caso del sitio I (Figura 6). 


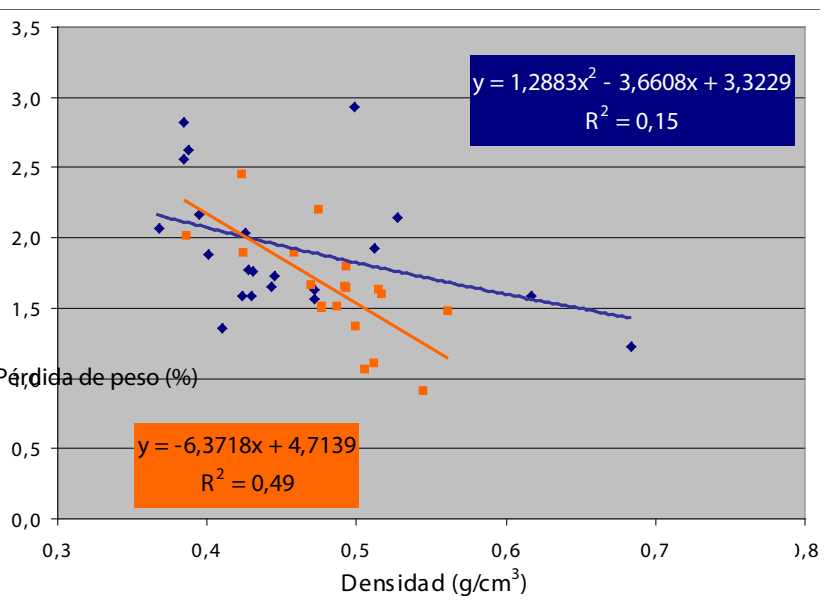

Figura 6. Ensayo de termites. Pérdidas de peso de los especímenes de madera en función de la densidad de la madera.

Tal como se indicó anteriormente, los ensayos del híbrido Populus y el duramen de Eucalyptus aún se están desarrollando.

\section{Ensayo de campo de estacas}

Este ensayo comenzó en julio de 2007 y la última evaluación se realizó a los 17 meses del comienzo del mismo.

Por el momento no se han detectado ataques de termites ni en el Eucalyptus ni en el Populus.

Se ha observado pudrición blanca no sólo en las estacas de Populus sino también en las de E. grandis. Por el momento no se ha detectado pudrición parda.

Las clasificaciones de Eucalyptus oscilaron entre 0 y 3, en donde 0 significa "sano" y 3 "ataque severo". La media total de los dos sitios y las dos posiciones fue 1,8 , en donde 1 corresponde a "ataque leve" y 2 indica "ataque moderado". Por otro lado, las clasificaciones de Populus variaron de 2 a 4, en donde 4 es la clasificación para "falla". El Populus presentó cuatro estacas falladas y un promedio de 3,2.

En la última evaluación, el sitio II presentó un desempeño superior al del sitio I. También se observó una mejor condición en los especímenes de $\mathrm{C}$ que en los de $\mathrm{A}$ (Figuras 7 y 8).

El sitio I y el sitio II presentaron clasificaciones de descomposición similares.

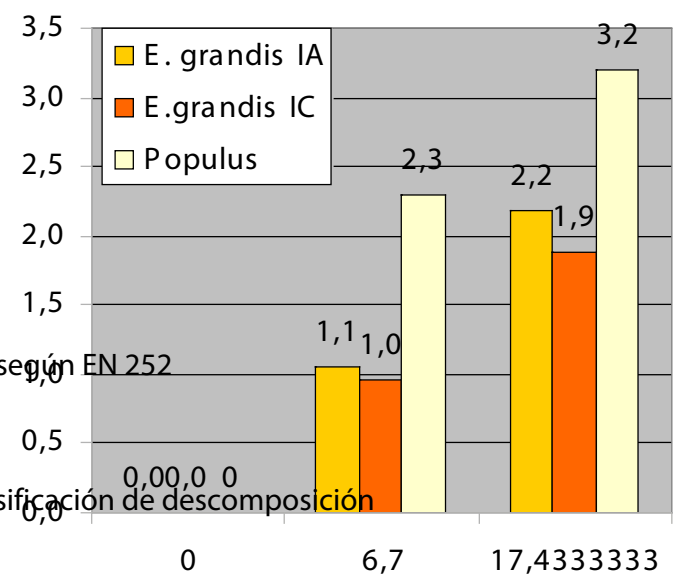

Tiempo (meses)

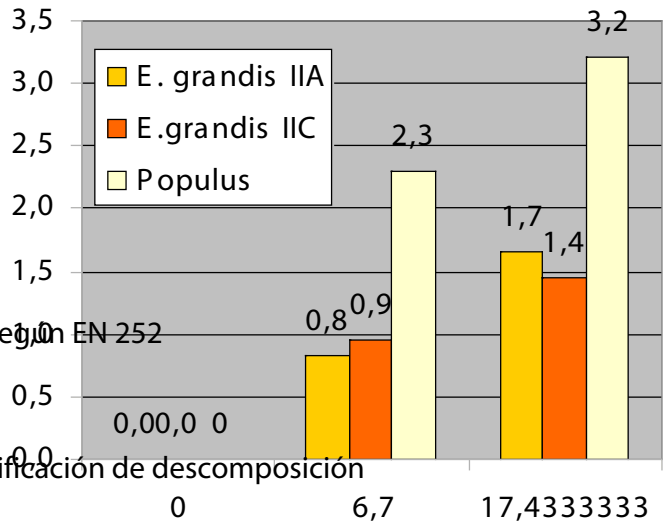

Tiempo (meses)

Figura 8. Clasificaciones promedio según EN 252 para el ensayo de campo de estacas. Sitio II.

\section{Ensayo de doble capa}

Esta prueba comenzó el mismo año que el ensayo de estacas.

Luego de transcurridos seis meses de instalado, pudo observarse pudrición blanca en el ensayo. Se extendió desde las vigas de apoyo de pino que ya estaban podridas. En ese momento se cambiaron los apoyos por vigas de Eucalyptus. En la segunda evaluación se pudo observar cierta descomposición en el punto de contacto entre ambas capas, que había estado funcionando como colector de agua, tal como se esperaba. La capa inferior presentó siempre un peor estado de descomposición. Hasta la última evaluación, la pudrición blanca fue el único tipo de pudrición observada.

Durante los 17 meses de la prueba, el E. grandis arrojó mejores resultados que el híbrido Populus (Figuras 9 y 10).

Ambas posiciones, A y C, presentan las mismas clasificaciones promedio. Tampoco es posible diferenciar los sitios II y I entre sí.

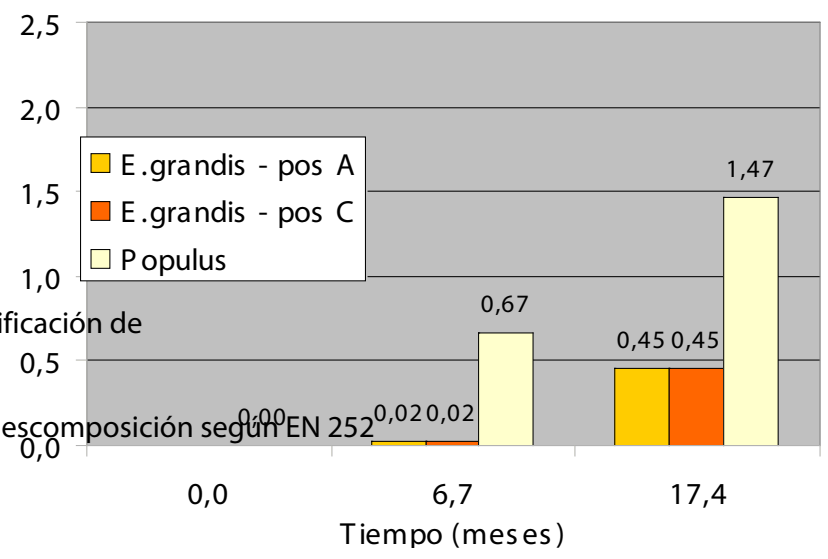

Figura 9. Clasificaciones según EN 252 para el ensayo de doble capa. Sitio I. 


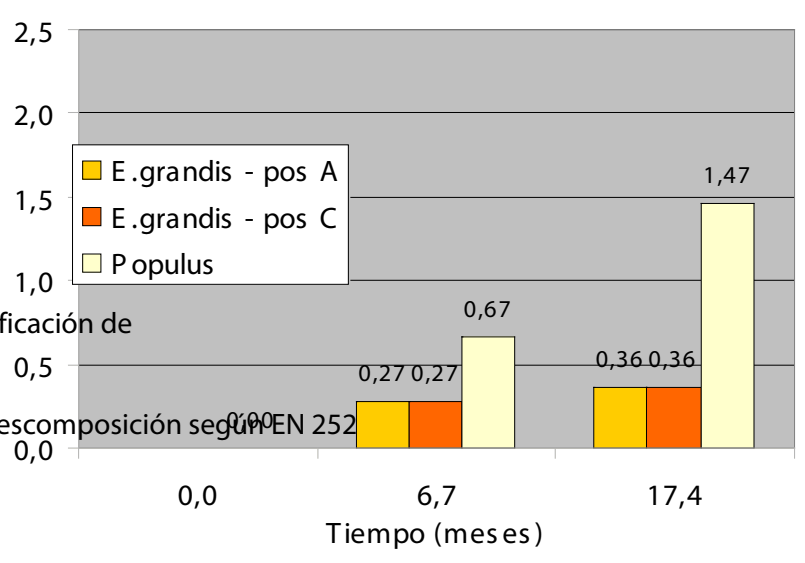

Figura 10. Clasificaciones según EN 252 para el ensayo de doble capa. Sitio II.

\section{Conclusiones}

Las conclusiones que se expresan a continuación se refieren a las plantaciones de rápido crecimiento de E. grandis de 16 años de edad muestreadas en la región norte (sitio I) y oeste (sitio II) de Uruguay.

1. El duramen de E. grandis que se analiza en el presente no sería madera adulta. El material de la posición A es madera juvenil en su totalidad, mientras que puede suponerse que el de la posición $\mathrm{C}$ es madera de transición. A partir de esto se concluye que las propiedades de la madera adulta podrían ser diferentes de las que aquí se informaron para la posición $\mathrm{C}$.

2. En todos los ensayos realizados, el duramen de E. grandis presentó una mayor durabilidad que el híbrido Populus.

3. Según EN 350-1, la madera juvenil de E. grandis puede clasificarse como "moderadamente durable" ante el hongo de pudrición parda G. trabeum, mientras que la madera de transición puede describirse como "durable" frente al mismo hongo.

4. La albura de Eucalyptus y el Populus presentaron la misma resistencia ante el G. trabeum.

5. Los ensayos de laboratorio con Trametes versicolor y Serpula lacrymans deberían ser repetidos.

6. En este trabajo, la madera de E. grandis fue atacada de forma moderada o grave por Reticulitermes spp.

7.El sitio de la plantación parece no afectar la vulnerabilidad a las termites del E. grandis cultivado en las regiones norte u oeste de Uruguay.

8. El desempeño en ensayos de campo de la madera proveniente del sitio II se vislumbra preliminarmente mejor que el del material del sitio I. Sin embargo, es aún muy pronto para extraer conclusiones sobre la durabilidad, ya sea del ensayo de doble capa o del de campo de estacas.

9. La madera de transición ha presentado mayor resistencia al hongo de pudrición parda Gloeophyllum trabeum, una susceptibilidad levemente menor a las termites y mejores condiciones en el campo luego de 17 meses de exposición. En base a estos resultados puede esperarse que la madera adulta de Eucalyptus grandis tenga mayor durabilidad natural que la madera juvenil.

10.Es posible que la durabilidad natural esté relacionada con la densidad. Deberían realizarse más estudios para obtener conclusiones sólidas.

\section{Reconocimientos}

Agradecemos a las personas y organizaciones que figuran a continuación:

- Al LATU y al gobierno uruguayo por el apoyo financiero al proyecto $\mathrm{PDT} / \mathrm{S} / \mathrm{C} / \mathrm{OP} / 35-11$ en el marco del PDT.

- A las empresas forestales Eufores e Industrias Forestales Puerto Arazatí, y al INIA (Instituto Nacional de Investigaciones
Agropecuarias), por brindarnos material sobre la madera.

- A la Dra. Ina Stephan, BAM.

- Al Dr. Koichi Yamamoto (FFPRI, Tsukuba, Japón), por el asesoramiento técnico.

- Al Dr. Holger Militz, por la estadía de Silvia Böthig en el Instituto de Biología de la Madera y Tecnología de la Madera, Universidad de Goettingen.

- Al Dr. Sadaaki Ohta, LATU, por su apoyo.

- A nuestro equipo de trabajo y a todas las personas que contribuyeron con este trabajo.

\section{Referencias}

\section{- AMERICAN WOOD PRESERVER'S ASSOCIATION STANDARD} (United States). E14-02: Standard method of evaluating wood preservatives in a soil bed. Birmingham: AWPA Subcomitee P-6, 2003.

- ASOCIACIÓN ESPAÑOLA DE NORMALIZACIÓN Y

CERTIFICACIÓN (España). UNE-EN 460: Durabilidad de la madera $y$ de los materiales derivados de la madera. Durabilidad natural de la madera maciza. Guía de especificaciones de durabilidad natural de la madera para su utilización según las clases de riesgo. Madrid: AENOR, 1995.

- ASOCIACIÓN ESPAÑOLA DE NORMALIZACIÓN Y CERTIFICACIÓN (España). UNE-EN 350-2: Durabilidad de la madera y de los materiales derivados de la madera. Durabilidad natural de la madera maciza. Parte 2: Guía de la durabilidad natural de la impregnabilidad de especies de madera seleccionadas por su importancia en Europa. Madrid: AENOR, 1995.

- BÖTHIG, S. Densidad y otros indicadores de calidad de Eucalyptus grandis para el mejoramiento genético enfocado a la calidad. En: IUFRO. Actas del Simposio. Desarrollando el Eucalipto del futuro. Tema 4: Mejoramiento para Calidad de Madera y Fibra, (Valdivia 1015 de setiembre 2001). Valdivia: IUFRO, 2001.

- DA SILVA OLIVEIRA, J.T. Problemas e oportunidades com a utilização da madeira de eucalipto. En: Universidad Federal de Vicosa. Workshop: Técnicas de abate, processamento e utilização da madeira de eucalipto, (Vicosa 22-24 de junio de 1999). Vicosa: Universidad Federal de Vicosa, 1999. p. 39-52.

- DOLDÁN, J. Indicadores de calidad de madera Eucalyptus grandis de Río Negro, Uruguay: contenido de humedad, densidad, contracción y largo de fibras. Montevideo: LATU, 2001. (Informe de Investigación. Serie Forestales; 9)

- DOLDÁN, J.; BÖTHIG, S. Propiedades físicas fundamentales en maderas de especies de prioridad forestal. En: JICA; LATU. Seminario de productos forestales, (Montevideo diciembre de 2002). Montevideo: LATU, 2002.

- DOWNES, G.M., et al. Sampling plantation eucalyptus for wood and fiber properties. Melbourne: CSIRO Publishing, 1997.

- EUROPEAN COMITEE FOR STANDARDIZATION (Belgium). EN 113: Wood preservatives. Test method for determining to protective effectiveness against wood destroying basidomycetes. Determination of toxic values. Brussels: ECS, 1996.

- EUROPEAN COMITEE FOR STANDARDIZATION (Belgium). EN 118: Wood preservatives. determination of preventive effectiveness against Reticulitermes santonensis de Feytaud. Brussels: ECS, 1990.

- EUROPEAN COMITEE FOR STANDARDIZATION (Belgium). EN $252 \mathrm{AC} 1$ : Field test method for determining the relative effectiveness of wood preservative in ground contact. Brussels: ECS, 1989.

- EUROPEAN COMITEE FOR STANDARDIZATION (Belgium). EN 350-1: Durability of wood and wood based products. Natural durability of solid wood. Part I: Guide to the principles of testing and classification of the natural durability of wood. Brussels: ECS, 1994.

- LORENZO, D., et al. Study of the natural durability of spanish Eucalyptus globulus wood [En línea]. En: IRG. $38^{\text {th }}$ Annual Conference of the International Research Group on Wood Preservation. Section 1. Biology, (Wyoming junio 2007). [Consulta: 25 de setiembre de 2008]. Disponible en: http://www.irg38.com/PDF\%20Presentations/0710617\%20Lorenzo\%20Troya.pdf

- MALAN, F. S. South Africa's experience. En: CTIA ; IUFRO. Proceedings of the CTIA/IUFRO International Wood Quality Workshop, (Quebec 18-22 de agosto de 1997). Quebec: IUFRO-CTIA, 
1997.

- MALAN, F.S. Variation, association and inheritance of juvenile wood properties of Eucalyptus grandis Hill ex Maiden with special reference to the effect of rate of growth. En: South African Forestry Journal. 1991, (157):16-23.

- NAGANO, Y. Fungus cellar testing as an evaluation method for performance of treated timber in ground. Stockholm: International Research Group on Wood Preservation, 2001. (IRG/WP; 01-20227).

- O’NEILL, H.; TARIGO, F.; IRAOLA, P. Propiedades mecánicas de Eucalyptus grandis Hill ex Maiden del Norte de Uruguay. Montevideo: LATU, 2005a. (Informe; 4).

- O'NEILL, H., et al. Propiedades mecánicas de Eucalyptus grandis Hill ex Maiden. del Norte de Uruguay. Montevideo: LATU, 2005b. (Informe; 5).

- O’NEILL, H., et al. Propiedades mecánicas de Eucalyptus grandis Hill ex Maiden. del Centro de Uruguay. Montevideo: LATU, 2005c. (Informe; 6).

- PÉREZ DEL CASTILlo, A. Propiedades mecánicas y calidad de madera de Eucalyptus grandis del norte de Uruguay. Montevideo: LATU, 2001. (Informe de Investigación. Serie Forestales; 4).

- RAPP, A. O.; AUGUSTA, U.; PEEK, R. D. Facts and ideas of testing wood durability above ground [En línea]. Hamburgo: BFH, 2001. [Consulta: 25 de setiembre de 2008]. Disponible en: http://www.bfafh.de/inst4/43/pdf/3doublay.pdf

- RAPP, A. O.; AUGUSTA, U. Some fundamental thoughts on the prediction of field performance from lab-testing based on comparison of lab and field results of some copper-free materials. En: IRG. $34^{\text {th }}$ Annual Meeting of the International Research Group on Wood Preservation. Section 2. Test Methodology and Assessment, (Brisbane 18-23 de mayo de 2003a). Brisbane: IRG, 2003.

- RAPP, A. O.; AUGUSTA, U. The natural durability of wood in different use classes. En: IRG. $34^{\text {th }}$ Annual Meeting of the International Research Group on Wood Preservation. Part I, Section 1. Biology, (Brisbane 18-23 de mayo de 2003b). Brisbane: IRG, 2003.

- RAPP, A. O., AUGUSTA, U., BRANDT, K. The natural durability of wood in different use classes. En: IRG. $37^{\text {th }}$ Annual Meeting of the International Research Group on Wood Protection. Section 1. Biology, (Tromsoe 18-22 de junio de 2006). Tromsoe: IRG, 2006.

- VAN ACKER, J., et al. Biological durability of wood in relation to end-use. Part 1. Towards a European standard for laboratory testing of the biological durability of wood. En: Holz als Roh-und Werkstoff. 2003, (61):35-45. 